Check for updates

Cite this: RSC Adv., 2017, 7, 22468

Received 20th February 2017

Accepted 13th April 2017

DOI: $10.1039 / \mathrm{c} 7 \mathrm{ra02084h}$

rsc.li/rsc-advances

\section{Lymphoma cell isolation using multifunctional magnetic nanoparticles: antibody conjugation and characterization $\uparrow$}

\author{
Soubhagya Laxmi Sahoo, ${ }^{a}$ Chi-Hsien Liu (iD *abcd and Wei-Chi Wu de
}

The early detection of B-cell lymphoma and non-Hodgkin's lymphoma has a wide impact on the diagnosis and therapy of lymphoma patients. Capturing and sorting tumour cells with magnetic nanoparticles (MNPs) has received considerable attention in recent years. Despite these successes, the efficient isolation of circulating tumour cells from complex biological fluids is still under development for the early diagnosis of lymphoma. In this study, MNPs are functionalized with anti-CD20 antibodies using an avidin-biotin linkage, with the aim of achieving specific cancer cell detection and efficient isolation. Anti-CD20 antibody-conjugated MNPs (Ab MNPs) could specifically target CD20-expressing lymphoma cells. The capture efficiency of the Ab MNPs in the lymphoma cell line was $>95 \%$ with regard to the mixture of two cell lines, as confirmed by flow cytometry and confocal microscopy. Transmission electron microscopy confirmed that the conjugation of an antibody with the MNPs increased the size from 12 to $47 \mathrm{~nm}$. The surface charge of the Ab MNPs was examined by using zeta potential measurements. Furthermore, Prussian blue staining was performed to confirm the interaction of Ab MNPs with the targeted lymphoma cells. Our results indicated that the receptor recognition ability of the antibody was fully retained after conjugation with MNPs. In conclusion, anti-CD20 MNPs can be used for very sensitive detection and quick isolation of CD20-positive lymphoma cells among mixed cells by using only a permanent magnet.

\section{Introduction}

Cancers are among the most serious diseases that can ultimately lead to death. Sensitive and rapid isolation of cancer cells from complex bio-fluids is of critical importance for cancer research, prevention and therapy. ${ }^{1}$ Cell sorting is often used to enrich rare cells for further well-defined culture conditions and to enhance the cell population. The current capture techniques for cancer cells include flow cytometry, magnetic-based sorting devices and microfluidic chips, using for example fluorescence signals, magnetic forces and physical principles. ${ }^{2}$ Flow cytometry provides precise isolation; however, it involves a sophisticated instrument and needs expensive fluorescent probes for

\footnotetext{
${ }^{a}$ Graduate Institute of Biochemical and Biomedical Engineering, Chang Gung University, 259, Wen-Hwa First Road, Kwei-Shan, Tao-Yuan 333, Taiwan

${ }^{b}$ Research Center for Chinese Herbal Medicine, Research Center for Food and Cosmetic Safety, College of Human Ecology, Chang Gung University of Science and Technology, 261, Wen-Hwa First Road, Taoyuan, Taiwan

${ }^{c}$ Department of Chemical Engineering, Ming Chi University of Technology, 84, GungJuan Road, New Taipei City, Taiwan

${ }^{d}$ Department of Ophthalmology, Chang Gung Memorial Hospital, 5, Fu-Hsing Street, Taoyuan, Taiwan

${ }^{e}$ College of Medicine, Chang Gung University, 259, Wen-Hwa First Road, Taoyuan, Taiwan.E-mail: CHL@mail.cgu.edu.tw
}

$\dagger$ Electronic supplementary information (ESI) available. See DOI: $10.1039 / \mathrm{c} 7 \mathrm{ra02084h}$ cell labelling. A robust sorting platform is needed to isolate tumour cells for further diagnosis and expansion. Among these techniques, magnetic sorting utilizes targeting magnetic nanoparticles (MNPs), which are biodegradable and have low toxicity. ${ }^{3,4}$ The MNP-based technology has several advantages, such as a high surface-to-volume ratio, high-binding capacity and specific interactions between nanoparticles. ${ }^{5}$ Moreover, the diffusion limitation within the micron-sized particles leads to a decrease in their binding efficacy on the targeted cells in biological fluids, such as blood. ${ }^{6}$ Therefore, the nano-scaled MNPs provide several advantages for capture applications, including a low diffusion barrier, high surface area, stability and specificity.

Specific targeting is a key step in realizing the full potential of MNPs in tumour-associated diagnosis and the capture of tumour cells. Researchers have devoted a tremendous amount of time to develop MNPs-based models for the capture and early detection of cancer cells that simultaneously conjugate MNPs to active targeting moieties, such as ligands and monoclonal antibodies. Antibodies are promising moieties for targeting cancer cells, using high affinity and ligand-receptor specificity for the surface antigen on the tumour. ${ }^{7}$ For example, the antiCD20 antibody (rituximab) has been applied to the treatment of non-Hodgkin's lymphoma and inflammatory diseases, such as rheumatoid arthritis and myositis. ${ }^{8}$ The CD20 is a nonglycosylated antigen expressed on B-cell non-Hodgkin's 
lymphomas, ${ }^{9}$ and cannot be found on stem cells, pro-B cells, normal plasma cells or other normal tissues. ${ }^{10}$ In addition, the CD20 on the cell membrane, which is not internalized in response to antibody binding, is a good candidate as a target for cell isolation.

Cancer cells have been successfully detected and isolated by using MNPs based on antibody and antigen interactions. ${ }^{11,12}$ Cirstoiu-Hapca et al. (2007) and Xu et al. (2014) have used antiHER2 and anti-GD2 antibodies conjugated with nanoparticles to specifically isolate the tumour cells bearing the surface antigens. ${ }^{6,13}$ However, only one type of cancer cell was isolated in their targeting studies. In contrast, Song et al. (2011) analyzed the capture efficiencies of tumour cells by using fluorescent MNPs to isolate a small number of spiked tumour cells in a large population of normal cells. ${ }^{14}$ Magnetic particles labelled with a fluorescent dye for optical detection and conjugated with a monoclonal antibody against the neu receptor have been demonstrated to significantly identify primary and metastatic breast tumours. ${ }^{15}$ The anti-EGFR antibody-conjugated nanoparticles can be used to capture circulating tumour cells expressing EGFR and in the subsequent diagnostic analysis. ${ }^{16} \mathrm{Wu}$ et al. have successfully developed multifunctional magnetic particles conjugated with anti-EpCAM antibody that could detect endogenous metabolites and isolate rare tumour cell isolation. ${ }^{17}$ These antibody-conjugated nanoparticles have been proven useful for sensitive detection and rapid isolation of cancer cells in early diagnoses.

Our objectives are to synthesize and characterize the antibody-conjugated MNPs (Ab MNPs) and to assay their biocompatibility and separation efficacy. The anti-CD20 Ab MNPs were synthesized to detect and isolate lymphoma cells from two kinds of mixed cells via a process based on the high affinity between antigens and antibodies. Specifically, the carboxylic group of MNPs was activated using the EDC/NHS linker, and then avidin was conjugated onto MNPs to form avidin MNPs. Biotin maleimide was conjugated with an antiCD20 antibody, and in the final step, a biotinylated antibody was added to interact with the avidin MNPs to form Ab MNPs. The morphology and surface charge of the Ab MNPs were examined with a transmission electron microscope and using zeta potential measurements. The use of Ab MNPs to specifically isolate the cancer cells was evaluated by flow cytometry analysis, confocal image and Prussian blue staining.

\section{Experimental section}

\section{Materials}

All the chemical reagents were commercially purchased and used without further purification. The materials include avidin, biotin maleimide, ethylenediaminetetraacetic acid (EDTA), Hoechst 33342, neutral red, $N$-(3-dimethylaminopropyl)-ethyl carbodiimide (EDC), hydroxy succinimide (NHS), and Trypan blue solution were purchased from Sigma-Aldrich (MO, USA). Rhodamine B was purchased from Acros Organics (NJ, USA). Potassium thiocyanate and hydrochloric acid were purchased from J.T Baker (PA, USA). Fetal bovine serum (FBS) was purchased from Biological Industries (Haemek, Israel). Paramagnetic iron oxide nanoparticles (COOH-surface modified) were purchased from Taiwan Advance Nanotech (Taoyuan, Taiwan).

\section{Functionalization of Ab MNPs}

For the targeting approach, the carboxylic group of magnetic nanoparticles $\left(1 \mathrm{mg} \mathrm{mL}^{-1}\right)$ were activated using EDC/NHS linker (0.64 and $1.2 \mathrm{mg} \mathrm{mL}^{-1}$, respectively) at a constant vortex rate for 2 hours at room temperature. Next, avidin $\left(0.2 \mathrm{mg} \mathrm{mL}^{-1}\right)$ was conjugated onto magnetic nanoparticles for 1 hour at room temperature to form avidin MNPs. The unconjugated avidin was removed by washing three times with deionized water using magnetic separation system (Millipore). The anti-CD20 antibodies were purified from the spent media of BCRC 60427 hybridoma cell line by using liquid chromatography and protein A sepharose column (GE). Separately, the biotinylated Ab was prepared as

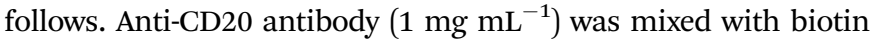
maleimide $\left(0.25 \mathrm{mg} \mathrm{mL} \mathrm{mL}^{-1}\right)$ in phosphate buffer $(\mathrm{pH} 7.2)$ and reacted for 2 hours at room temperature. The detailed methods are referred to the chemical conjugation textbook. ${ }^{18}$ In the final step, the biotinylated Ab was mixed with avidin MNPs to form the Ab MNPs for 30 minutes. The Ab MNPs was washed three times to remove unreacted biotinylated $\mathrm{Ab}$ and then stored at $4{ }^{\circ} \mathrm{C}$. For the dose effect of MNP isolation, high Ab conjugated MNPs $(15.86 \mu \mathrm{g}$ $\mathrm{Ab}$ per mg MNPs) and low Ab conjugated MNPs (8.07 $\mu \mathrm{g}$ Ab per mg MNPs) were prepared by adjusting the amount of biotinylated antibody in the conjugation procedure.

\section{Cell culture}

The hybridoma cells (BCRC 60427) secreting the immunoglobulin IgG2a, which recognizes the human CD20 antigen, Chinese hamster ovary cell line (CHO, BCRC 60185), and CD20expressing Ramos lymphoma (BCRC 60252) were obtained from the Bioresource Collection and Research Center (BCRC, Hsinchu, Taiwan). BCRC 60252 was maintained in RPMI-1640 medium supplemented with $10 \%$ heat inactivated fetal bovine serum (FBS). BCRC 60427 was maintained in CD hybridoma medium (Thermo Fisher Scientific, Sugar Land, USA). The CD20 free cell line such as CHO BCRC 60185 was maintained in Excel medium. HaCat cells are kindly donated by Prof. Sheu, HammMing (NCKU, Taiwan) and maintained in DMEM with 10\% serum. All cells were incubated at $37{ }^{\circ} \mathrm{C}$ under a humidified atmosphere with $5 \% \mathrm{CO}_{2}$. The cell morphology and growth were monitored daily using a light microscope. Cell passage was performed every four days to maintain an exponential growth phase. The cell density and viability were determined using a Beckman Coulter counter (MS3 model) and hemocytometer, respectively, prior to all experiments.

\section{Characterization}

The size and surface property of MNPs were characterized by transmission electron microscopy (TEM). A drop of diluted sample was dispersed onto a 100-mesh copper grid (CF200-Cu, Electron Microscopy Science) and the excess drop was removed with filter paper. The sample containing copper grid was dried for 2 hours at $55{ }^{\circ} \mathrm{C}$ prior to TEM analysis. The morphology of the $\mathrm{COOH}$ MNPs and Ab MNPs were observed by TEM (JM-1011, 
JEOL, and Tokyo, Japan). The zeta potential was characterized using a Zetasizer Nano ZS 90 (Malvern, Worcestershire, U.K.) at $32{ }^{\circ} \mathrm{C}$. The tested MNPs under different $\mathrm{pH}$ conditions were $1 \mu \mathrm{g}$ $\mathrm{mL}^{-1}$. The different $\mathrm{pH}(\mathrm{pH} 3,5,7,9$, and 11) were obtained by adjusting the amount of HCL $(0.1 \%)$ and $\mathrm{NaOH}(0.1 \%)$. Fourier transform infrared spectroscopy (Alpha, Bruker, Germany) was used to characterize the presence of specific chemical groups in the modified magnetic nanoparticles. Dried samples $(0.8 \mathrm{mg})$ were mixed with $\mathrm{KBr}$ (IR grade, Sigma) powder (80 mg) and compressed into a thin membrane using a desktop pellet press (ICL, Garfield, NJ). The spectra of the samples were then processed by Bruker OPUS software. The loading antibody on the MNPs was determined by Nanodrop spectrophotometer (Thermo, Wilmington, DE).

\section{Detection and isolation of CD20 positive lymphocyte cells by Ab MNPs}

To demonstrate the abilities of Ab MNPs for detection and isolation of cancer cell, Ramos lymphoma cells were used as target cells. The CD20 free cells including hybridoma cells, HaCaT and CHO cells were used as control. The CD20 positive cells stained with Hoechst 33342 nucleic acid dye with a final concentration of $1 \mathrm{ppm}$ and the control cells were stained with Rhodamine B (with a final concentration of $10 \mathrm{ppm}$ ) were mixed with Ab MNPs or COOH MNPs and incubated for 20 minutes at $4{ }^{\circ} \mathrm{C}$ to prevent the endocytosis. The target cells bound by their prospective MNPs were washed three times with PBS using a magnet and then imaged with the aid of a confocal microscope and flow cytometry. To demonstrate the Ab MNPs selectivity towards the target cells, we carried out a control experiment with target cancer cells and non-target normal cells. We mixed $\mathrm{Ab}$ MNPs with a sample containing $1 \times 10^{6} \mathrm{CD} 20$ positive lymphoma cells and CD20 negative cells and incubated for 20 minutes at $4{ }^{\circ} \mathrm{C}$. After magnetic separation, the precipitate was imaged under a confocal microscope and flow cytometry. The number of cells before and after isolation with $\mathrm{Ab}$ MNPs was imaged with the confocal microscope to know the efficiency of the Ab MNPs to capture the target cancer cells.

\section{Targeting efficiency of Ab MNPs to lymphocyte cells}

Magnetic separation was performed by adding Ab MNPs to each $1 \mathrm{~mL}$ of sample $\left(1 \times 10^{6}\right.$ cells $)$ as describe in the above procedure. The different concentration $\left(10,30,50 \mu \mathrm{g} \mathrm{mL}{ }^{-1}\right)$ of high $\mathrm{Ab}$ conjugated MNPs (15.86 $\mu \mathrm{g}$ Ab per mg MNPs), and low Ab conjugated MNPs (8.07 $\mu \mathrm{g}$ Ab per mg MNPs) were incubated with the cell samples for 20 minutes at $4{ }^{\circ} \mathrm{C}$. Then, a magnet was introduced to the sample tubes, and after 3 minutes the target cells were attached to the tube wall while the supernatant was collected carefully using a pipet. After magnetic separation, the number of isolated cells by Ab MNPs was determined with flow cytometry to know the efficiency of the Ab MNPs to capture the target lymphocyte cells.

The specificity of Ab MNPs towards CD20 positive lymphocytes was evaluated by mixing target cells with CD20 free cells in different ratios. The Hoechst 33342 stained lymphocytes were varied from $5 \times 10^{4}$ to $1 \times 10^{6}$ and $\mathrm{n} \mathrm{CD20}$ free cells by stained Rhodamine B were fixed at $1 \times 10^{6}$. The mixed cells were incubated with the Ab MNPs $\left(30 \mu \mathrm{g} \mathrm{mL}^{-1}\right)$ for 20 minutes. The isolation was performed using a magnet and the isolated cells were analyzed by flow cytometry. The isolation efficiency of the Ab MNPs was calculated as follows.

$$
\begin{aligned}
& \text { Isolation efficiency }(\%)=(\text { isolated CD20 positive cells/initial } \\
& \text { CD20 positive cells }) \times 100
\end{aligned}
$$

\section{Biocompatibility studies}

The CD20-expressing cells and CHO cells were employed for cytotoxicity evaluation. Cells treated with Ab MNPs at different concentrations were examined with Coulter counter, MTT, and Trypan blue assay to measure cell viability. For MTT assay, cells were seeded into 96-well plates $\left(2 \times 10^{4}\right.$ cells per well) with 100 $\mu \mathrm{L}$ medium and incubated overnight. Subsequently, Ab MNPs at different concentrations $\left(10,30,50 \mu \mathrm{g} \mathrm{mL}{ }^{-1}\right)$ were added to the well and incubated for 24 hours at $37{ }^{\circ} \mathrm{C}$. The control group was incubated with only sterile PBS. Add $200 \mu \mathrm{L}$ of $0.5 \mathrm{mg} \mathrm{mL}^{-1}$ MTT reagent into each well and incubated for additional 4 hours. After incubation, the supernatants were removed carefully and $200 \mu \mathrm{L}$ of DMSO was added to each well. Next, plates were shaken on an orbital incubator for 10 minutes in order to dissolve the formazan crystals. Finally, the absorbance of each well was measured by a spectrophotometer. Also, coulter counter and Trypan blue assay was performed to count the cell number and to evaluate the toxicity effect. The cells $\left(2 \times 10^{5}\right.$ cells per well) were cultured in a 6 well plate. The control group were incubated with sterile PBS, whereas different concentrations $\left(10,30,50 \mu \mathrm{g} \mathrm{mL}^{-1}\right)$ of Ab MNPs were added to cells. At each hour, $100 \mu \mathrm{L}$ of the cell suspension was transferred to an Eppendorf, and the cell number was evaluated by using Beckman Coulter counter (MS3 model) and femocytometer. For all the experiments, measurements were carried out in triplicate. The viability of the cells in the treated groups was calculated according to the following equation.

Viability $(\%)=($ final cell population of treated group/final cell population of control group) $\times 100$

\section{Localization of nanoparticles in the lymphoma cell line}

Prussian blue staining and potassium thiocyanate method were used to study iron uptake in cells. Equal volumes of $10 \%$ potassium ferrocyanide solution and $20 \%$ hydrochloric acid solution were freshly mixed to prepare the Prussian blue solution. Ramos lymphoma cells $\left(1 \times 10^{6}\right)$ were incubated with $30 \mu \mathrm{g} \mathrm{mL}{ }^{-1} \mathrm{Ab}$ MNPs or COOH MNPs for one hour at room temperature. Then, washed three times with PBS and incubated 5 minutes with $150 \mu \mathrm{L}$ ice-cold ethanol (95\%). After five minutes, cells were centrifuged to remove ethanol and washed three times with deionized water. Then, the cells were incubated with $150 \mu \mathrm{L}$ of Prussian blue solution for 20 minutes in the dark. After washing three times in PBS, the cells were counterstained with neutral red ( $1 \mathrm{ppm})$ for 2 minutes and imaged under the microscope for Prussian blue staining. 
For potassium thiocyanate method, $1 \times 10^{6}$ cells from Ramos lymphoma cell line, hybridoma or CHO cells were incubated with $30 \mu \mathrm{g} \mathrm{mL}{ }^{-1} \mathrm{MNPs}$ at $37{ }^{\circ} \mathrm{C}$ for 2 hours. The excess iron was removed by washing with $\mathrm{PBS}$, and the iron concentration in $1 \times$ $10^{6}$ cells was determined using the following thiocyanate-based spectrophotometric assay. The cell samples were mixed with 100 $\mu \mathrm{L}$ of $12 \mathrm{~N} \mathrm{HCl}$ for 4 hours at room temperature. Then, $400 \mu \mathrm{L}$ of $5 \%(\mathrm{w} / \mathrm{v})$ potassium thiocyanate was added to the solution and incubated for 15 minutes. Samples were centrifuged at $12000 \times g$ for $10 \mathrm{~min}$ and $100 \mu \mathrm{L}$ of the supernatant was added to a 96 well microtiter plate. The absorbance at $480 \mathrm{~nm}$ was measured using a microplate reader (Synergy HT, BioTek, Hong Kong). Two calibration curves of MNPs were prepared by using Ab MNPs and $\mathrm{COOH}$ MNPs (antibody free). The detailed procedure of potassium thiocyanate method was referred to previous papers. ${ }^{\mathbf{1 9 , 2 0}}$

\section{Results and discussion}

\section{Functionalization of antibody magnetic nanoparticles (Ab MNPs)}

We firstly chose biotin maleimide to conjugate the biotins to antibodies. The maleimide-containing biotins efficiently react to the thiol groups on the antibody and form a thio-succinimide linkage that can maintain the antibody's targeting ability. The disulfide bonds of antibodies as conjugation sites provide advantages over other reactive groups, such as amines and carboxylates. Avidin is conjugated with $\mathrm{COOH}$ MNPs through the EDC/NHS linker, and the biotinylated antibody is eventually bound with $\mathrm{COOH}$ MNPs through a biotin-avidin non-covalent interaction to form Ab MNPs. The strong binding affinity of biotin towards avidin is stable during proteolysis and within a wide range of $\mathrm{pH}$ and temperature levels and with a variety of denaturing agents. ${ }^{\mathbf{1 4 2 1 - 2 3}}$ The EDC/NHS linker directly connects carboxylic and amino groups, for conjugating molecules with multiple carboxylic and amino groups. The functionalization procedure of Ab MNPs is shown in the schematic diagram (Fig. 1). The conjugation strategy allows the functional and directional conjugation of the Fc portion of an antibody to MNPs and is directed outward from the surfaces of Ab MNPs, while the Fab (capture site) is largely available for efficient targeting. Conjugating MNPs specifically to the Fc portion decreases the opsonin role of the antibody molecules that are used for targeting the cell surface antigen. ${ }^{24}$ While conjugating the Fc-directed method, the opsonin activity of the antibody is concealed and reduces the

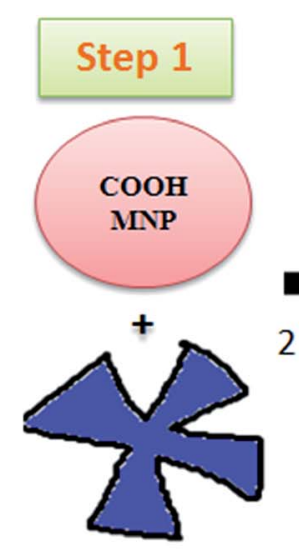

Avidin
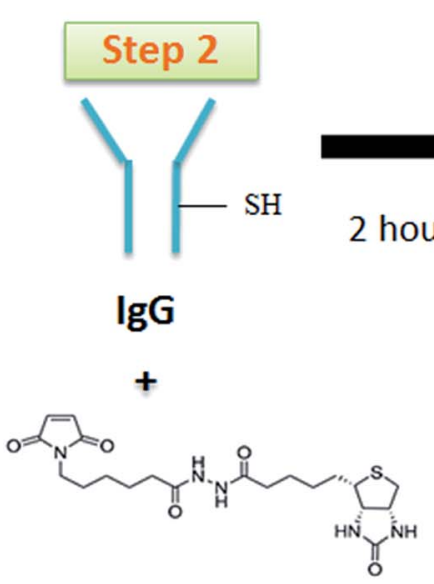

Step 3

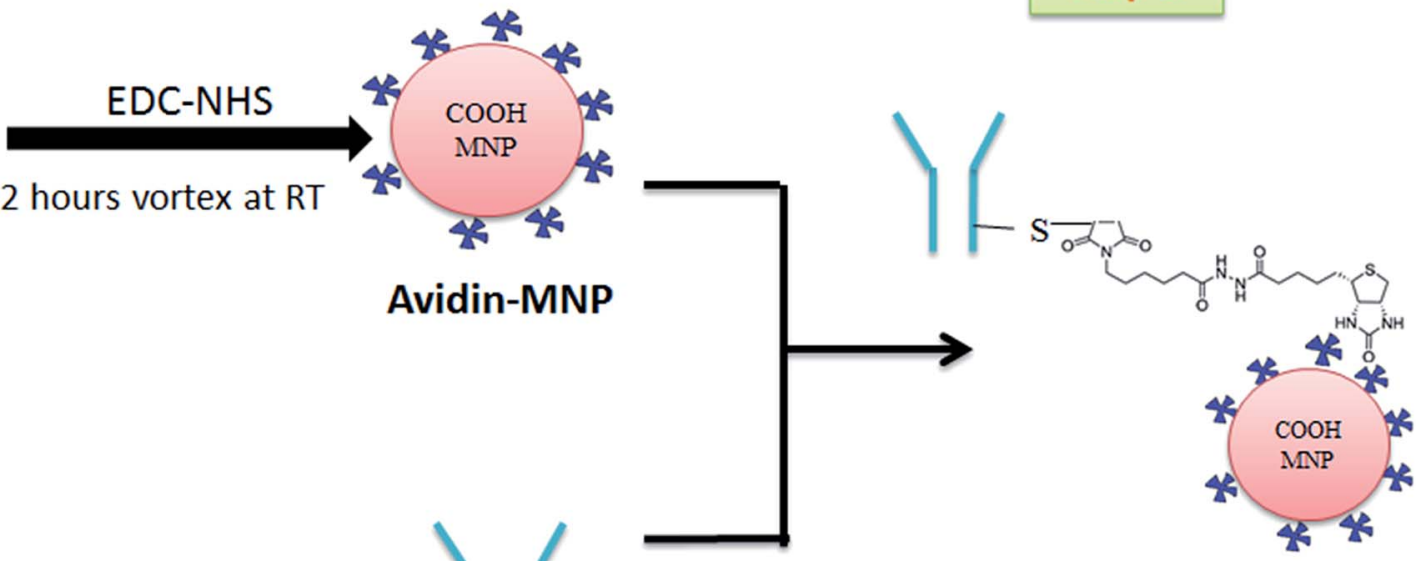

Ab MNPs

2 hours vortex at RT

\section{Biotinylated Ab}

\section{Biotin-maleimide}

Fig. 1 Schematic representation of antibody and MNPs conjugation. step 1. Avidin is conjugated with COOH MNPs in presence of EDC/NHS linker, step 2. Biotin is conjugated with the anti-CD20 antibody to obtain biotinylated antibody, step 3. Finally, biotinylated antibody is eventually bound with avidin modified $\mathrm{COOH}$ MNPs through a biotin-avidin non-covalent interaction to form antibody MNPs (Ab MNPs). 


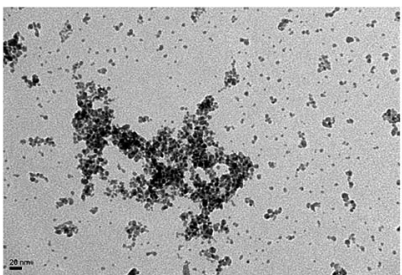

(a)



(b)
Fig. 2 TEM image for $\mathrm{COOH}$ MNPs (bare MNPs) and Ab MNPs (conjugated with $\mathrm{Ab}$ ). (a) $\mathrm{COOH}$ MNPs (b) modified Ab MNPs. The average sizes for $\mathrm{COOH}$ MNPs and Ab MNPs were $12 \mathrm{~nm}$ and $47 \mathrm{~nm}$ respectively by using image $\mathrm{J}$.

endocytosis of MNPs. Finally, modified MNPs with fewer cytotoxic effects are expected. The target efficiency of the anti-CD20 antibody in relation to the CD20 antigen result in functionalized $\mathrm{Ab}$ MNPs. The modified MNPs are characterized and investigated in terms of the isolation of lymphoma cells in the following sections.

\section{Characterization of Ab MNPs}

Physical properties were measured using TEM, zeta potential, and Prussian blue staining to confirm the quality of the $\mathrm{Ab}$ MNPs. The size and morphology of the Ab MNPs were investigated by TEM (Fig. 2). The particle size was calculated with at least 50 particles chosen at random in both the prepared samples through an image J analysis program. From Fig. 2(a and b), it is clear that the size of the MNPs increased from $12 \mathrm{~nm}$ to $47 \mathrm{~nm}$ after conjugation with $\mathrm{Ab}$. The properties of the coating materials have a major effect on the diameter, and the diameters of Ab MNPs were shown to be larger than the bare MNPs. Further, the thickness of the coating layer around the surface of the particle increased due to the decrease of water molecules in the magnetic core, which proves the successful conjugation of MNPs with Ab. The smaller size of the MNPs might improve their ability to enter the target site and to avoid endocytosis. Here, we have obtained Ab MNPs of about $47 \mathrm{~nm}$; in a previous study, the sizes of antibody-modified nanoparticles were 340 to $410 \mathrm{~nm} .^{25}$ The TEM images of the antibodyconjugated MNPs revealed a uniform size distribution and no significant change in their morphology, even after the conjugation reaction.

Fig. 3 illustrates the zeta-potential of $\mathrm{COOH}$ MNPs (unconjugated $\mathrm{Ab}$ ) and $\mathrm{Ab}$ MNPs (conjugated with $\mathrm{Ab}$ ) as a function of $\mathrm{pH}$, which has been shown to confirm the presence of functional groups on the surface of MNPs. The zeta potential values for $\mathrm{COOH}$ MNPs and $\mathrm{Ab}$ MNPs were 3 and $5 \mathrm{mV}$, respectively, at $\mathrm{pH}$ 3. The zeta potential of $\mathrm{COOH}$ MNPs was observed at around $-21 \mathrm{mV}$. However, the zeta potential of the Ab MNPs shifted from $-21 \mathrm{mV}$ to $-10 \mathrm{mV}$ at $\mathrm{pH} 11$ (Fig. 3), confirming the successful conjugation of $\mathrm{Ab}$ to the surface of the MNPs. The positive zeta potential values started to decrease for both the MNPs as the $\mathrm{pH}$ was raised towards 11 . The negative zeta values started to increase with the increase in $\mathrm{pH}$, which indicated that the surface charges of both conjugates had increased slightly. The fact that the isoelectric points of $\mathrm{COOH}$ MNPs and Ab MNPs

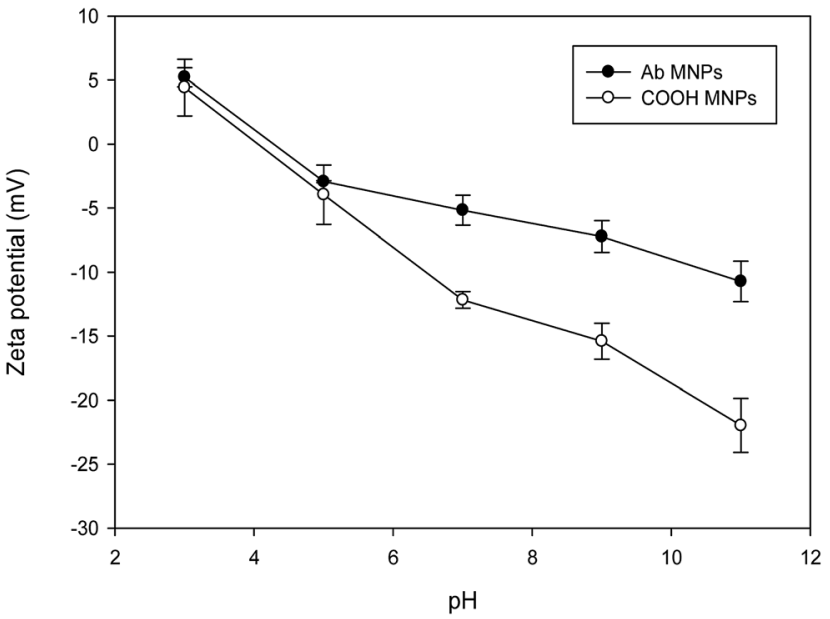

Fig. 3 Zeta potentials of $\mathrm{COOH}$ MNPs (unconjugated $\mathrm{Ab}$ ) and $\mathrm{Ab}$ MNPs (conjugated with $\mathrm{Ab}$ ) under different $\mathrm{pH}$ conditions. MNPs were dispersed in water ranged from $\mathrm{pH} 3$ to $\mathrm{pH} 11$.

were 3.6 and 4.4, respectively, was attributed to the presence of carboxylic groups in the MNPs. The IR spectra of COOH MNPs and Ab MNPs were presented in Fig. S1. $\dagger$ A prominent absorption band at $1717 \mathrm{~cm}^{-1}$ in $\mathrm{COOH}$ group (Fig. S1A $\dagger$ ) which was due to $\mathrm{C}=\mathrm{O}$ stretch of carboxylic group. On the other hand, in Ab MNPs (Fig. S1B $\dagger$ ), bands related to the presence of protein show up, the amide I and amide II bands at 1646 and 1533 $\mathrm{cm}^{-1}$, respectively. These results confirmed that the MNPs were successfully conjugated with antibodies.

\section{The capture and isolation of CD20-positive lymphocyte cells by Ab MNPs}

We developed a system in which the constituents are avidinlabelled MNPs, conjugated to a biotin-labelled anti-CD20 antibody (Ab MNPs) (Fig. 1). To demonstrate the specific capturing efficiency of $\mathrm{Ab}$ MNPs in targeting CD20-positive lymphocyte cells, we analyzed the MNP-targeted cells using confocal microscopy and flow cytometry. To examine the specific or nonspecific binding effect of MNPs, a magnetic isolation experiment was conducted, as described in the Experimental section. The 106 cells treated by $30 \mu \mathrm{g}$ of MNPs per mL were used for the cell capture assay. To test the capture efficiency, two kinds of cells (lymphoma and hybridoma cells) were mixed and incubated for 20 minutes with MNPs followed by isolation using a magnet. After magnetic isolation, the flow cytometry analysis indicated that $\mathrm{COOH}$ MNPs did not show specificity towards any cell lines. Sequentially, an equal number of mixed cells, that is, $48 \%$ of the hybridoma cells (CD20 negative cells) and $50 \%$ of the lymphocyte cells (CD20-positive cells) were obtained by COOH MNPs. In contrast, Ab MNPs separated 98\% of lymphocyte cells and a negligible percentage (only 2\%) of hybridoma cells (Fig. 4b). The fact that $\mathrm{COOH}$ MNPs could nonspecifically capture both cell types (Fig. S3†) from mixed cells indicated that $\mathrm{COOH}$ MNPs need further modification to enhance the selectivity. ${ }^{26}$ It also implies that the Ab MNPs display higher binding sensitivity than that of bare MNPs (COOH MNPs). More importantly, our conjugation strategy allows the quick and 


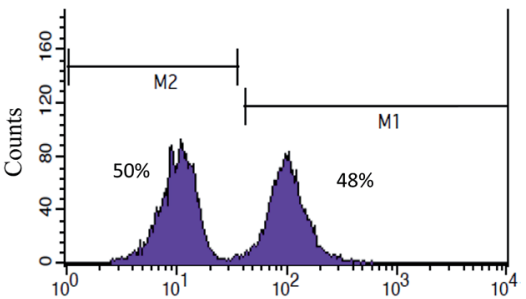

(a)

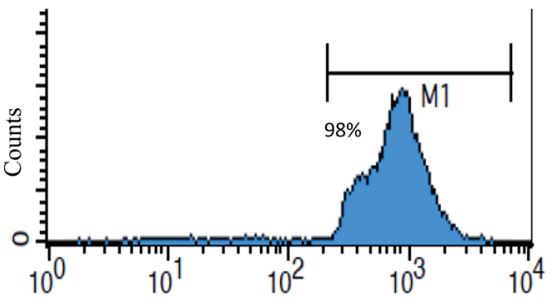

(b)

Fig. 4 Representative specific cell isolation using Ab MNPs or COOH MNPs. (a) Cell sorting by COOH MNPs and (b) cell sorting by Ab MNPs. Two kinds of cells (BCRC 60427 and Ramos lymphoma) were incubated with (a) COOH MNPs (50 $\mu \mathrm{L}$ ) or (b) Ab MNPs (50 $\mu \mathrm{L}$ ). The targeted cells were isolated with a magnet and washed three times. The results are expressed as the mean and standard deviation from two experiments, (a) $52 \% \pm$ 2.8 lymphoma cells and $46.3 \% \pm 2.3$ hybridoma cells were isolated by $\mathrm{COOH}$ MNPs. (b) $97.5 \% \pm 0.7$ lymphoma cells were isolated by Ab MNPs.

efficient capture of CD20-positive cells using a low dose of $\mathrm{Ab}$ MNPs. In a previous approach, more than an hour of incubation was required for the isolation of the cells. ${ }^{6}$ The specific binding capacity of Ab MNPs was made possible by targeting the anti$\mathrm{CD} 20 \mathrm{Ab}$ at the CD20 antigen on lymphocyte cells. These results suggested that the conjugation of anti-CD20 with MNPs can effectively enhance the specific binding of nanoparticles to the cells.

The ability of $\mathrm{Ab}$ MNPs to recognize the target cells was evaluated in the following experiments. The Ab MNPs were incubated with mixed cells (Fig. 4b), as mentioned in the Experimental section. For the specific isolation, the hybridoma cells (BCRC 60427) and HaCaT cells (skin cells) were used as the negative control and lymphocyte cells expressing $\mathrm{CD} 20$ were the target cells. Because the control cells had been stained with Rhodamine B and the target cancer cells with Hoechst dyes, they could be visualized by their different fluorescent qualities when excited under various levels of excitation. If blue fluorescence from the target cell nucleus and red fluorescence on the surface of the cells appeared, we could conclude that the respective cells were detected by the Ab MNPs. The cell mixtures were incubated with Ab MNPs for 20 minutes and isolated by a magnet. Confocal microscopic images of the precipitates were then taken. Fig. $\mathrm{S} 3 \dagger$ shows the experimental results in which the Ab MNPs isolated only CD20-positive lymphoma cells stained with blue fluorescence (from Hoechst 33342), while CD20-free cells stained with red fluorescence (Rhodamine B) were found in the supernatant (as presented in Fig. S4 in the ESI $\dagger$ section). $\mathrm{Ab}$ MNPs binding to CD20-negative cells (such as BCRC 60427 cells or HaCaT cells) was negligible, indicating the ability to recognize CD20 antigens with high specificity. The binding of Ab MNPs to CD20-positive cells was confirmed in the confocal images (Fig. S3†). For the recognition and isolation of markers on the cell surface, MNPs are advantageous because the high volume ratio offers a greater surface area for attaching specific ligands and for capturing markers. Most importantly, the size of the MNPs is smaller by orders of magnitude (approximately) than that of a cell, which allows multiple MNPs to be attached to a cell to facilitate magnetic isolation. Hence, the advantages of using the surface-modified MNPs with a target ligand for targeting and isolating cells are that they require only a short incubation time (20 minutes) and a simple washing process with a PBS buffer. The above experiments suggested that the mechanism of interaction between CD20-expressing cells and Ab MNPs is cell surface clustering. ${ }^{27}$ The high target specificity makes these MNPs ideal as isolation tools for recognizing and sorting cells.

After showing the expected binding, we tested the feasibility of Ab MNPs to isolate target cells from cell mixtures by first preparing mixed samples of CD20-positive cells (lymphoma) and CD20 negative cells, such as hybridoma cells (BCRC 60427), CHO (Chinese hamster ovary cells) and HaCaT cells. The

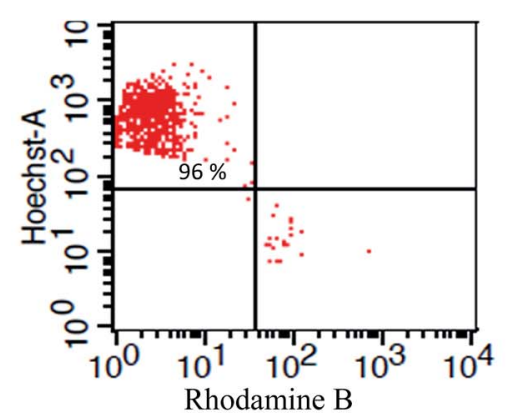

(a)



(b)

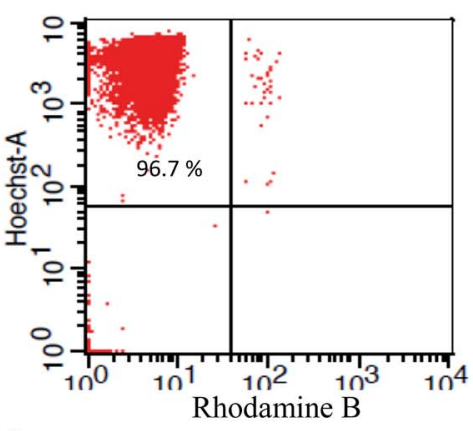

(c)

Fig. 5 Flow cytometric analysis for cell separation efficacy using Ab MNPs in different cell mixtures. (a) Lymphoma and hybridoma, (b) lymphoma and $\mathrm{CHO}$ cells and (c) lymphoma and $\mathrm{HaCaT} .30 \mu \mathrm{g} \mathrm{mL}{ }^{-1} \mathrm{Ab}$ MNPs were added to each cell samples and incubated for 20 minutes at $4{ }^{\circ} \mathrm{C}$. The results are obtained as the mean and standard deviation of two determinations, $96.5 \% \pm 0.7$ (a) $94.5 \% \pm 0.7$ (b) and $94.8 \pm 2.6$ (c). 
sample was prepared by mixing approximately $1 \times 10^{6}$ target cells and $1 \times 10^{6}$ of other kinds of cells, such as hybridoma, CHO or HaCaT cells, and then incubated with $30 \mu \mathrm{g} \mathrm{mL}^{-1}$ of $\mathrm{Ab}$ MNPs. The lymphoma cells were stained with Hoechst and BCRC 60427, and CHO or HaCaT cells were stained with Rhodamine $\mathrm{B}$ to distinguish the target and non-target cells. After incubation and magnetic separation, the magnetically isolated cells were analyzed by flow cytometry. When lymphoma cells mixed with CD20-negative hybridoma cells, the isolated cells contained $96 \%$ of CD20-positive cells, as shown in Fig. 5. Similarly, when mixed with either $\mathrm{CHO}$ or HaCaT cells, 95\% of CD20 positive cells could be isolated by using the Ab MNPs. We also examined the cells that remained in the supernatant, and most of these cells were CD20 negative, as shown in Fig. S4. $\dagger$

\section{Dose effect of Ab MNPs on the isolation of target lymphoma cells}

To evaluate the effects of different concentrations, different doses of antibody were conjugated with MNPs to isolate CD20positive cells and quantified by flow cytometry. A comparison of the high Ab-loaded MNPs (15.86 $\mu \mathrm{g}$ Ab per mg MNPs) and the low Ab-loaded MNPs (8.07 $\mu \mathrm{g}$ Ab per mg MNPs) was evaluated with mixed cells having both CD20-positive cells and CD20negative cells. Different amounts $\left(10,30\right.$, and $\left.50 \mu \mathrm{g} \mathrm{mL} \mathrm{m}^{-1}\right)$ of both high and low Ab-loaded MNPs were incubated with lymphoma (CD20 positive) and CD20-negative cells for 20 minutes and isolated by 3 minutes of magnetic separation. The CD20-positive cell binding increased with increasing concentrations of Ab MNPs. Both the high and low Ab-loaded MNPs showed good separation ability (Fig. 6a-f). The high Ab-loaded MNPs $\left(10 \mu \mathrm{g} \mathrm{mL}^{-1}\right)$ could efficiently isolate $95 \%$ of the CD20positive cells from CD20-negative hybridoma cells. Furthermore, $30 \mu \mathrm{g}$ and $50 \mu \mathrm{g} \mathrm{mL} \mathrm{m}^{-1}$ of Ab MNPs separated $96 \%$ and $98 \%$ of CD20-positive cells, respectively. In contrast, the low Abloaded MNPs showed that $10 \mu \mathrm{g} \mathrm{mL}{ }^{-1}$ of MNPs separated $88 \%$ of CD20-positive cells (Fig. 6d). The cell-capture rate of high concentrations of Ab MNPs had higher separation efficacy even at a low concentration $\left(10 \mu \mathrm{g} \mathrm{mL} \mathrm{m}^{-1}\right)$ of $\mathrm{Ab} \mathrm{MNP}$ treatment of cells. Whereas, with a low concentration of Ab MNPs, at $10 \mu \mathrm{g}$, $30 \mu \mathrm{g}$ and $50 \mu \mathrm{g} \mathrm{mL}{ }^{-1}$, separated $88 \%, 90 \%$ and $95 \%$ of CD20positive cells, respectively, and resulted in a few more CD20 negative cells compared with the high Ab MNPs (Fig. 6a-f). This proves the successful $\mathrm{Ab}$ conjugation on MNPs since lower concentrations of Ab MNPs can specifically bind to CD20positive cells. The isolation of the CD20 cells demonstrated some differences in binding, further indicating the Ab effect on MNPs. However, in the studied concentration range, Ab MNPs with high $\mathrm{Ab}$ conjugation exhibited more specific isolation of lymphocyte cells than those with low Ab modification on MNPs. A similar result was obtained when CD20-positive cells were mixed with CD20-negative CHO cells, as indicated in Fig. S7.† In a previous report, it was suggested that an optimal

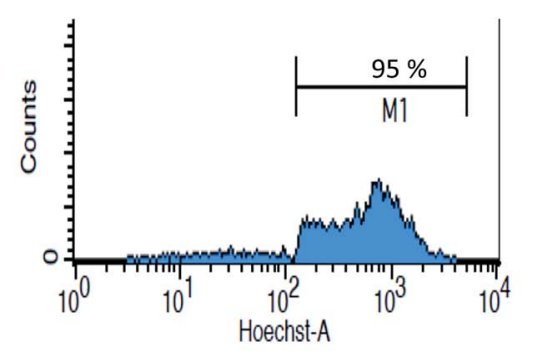

(a)

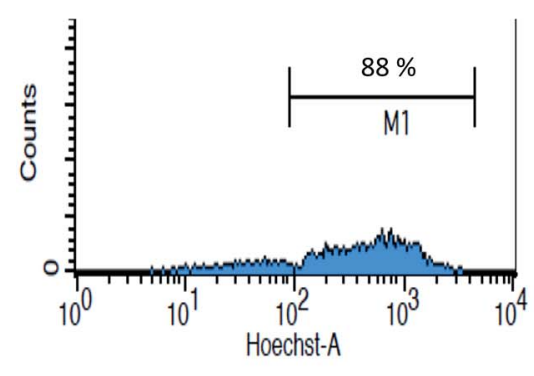

(d)



(b)

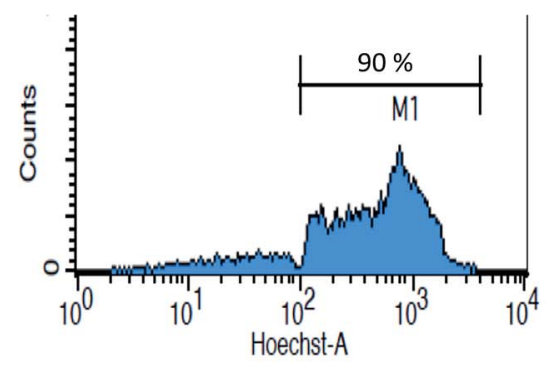

(e)

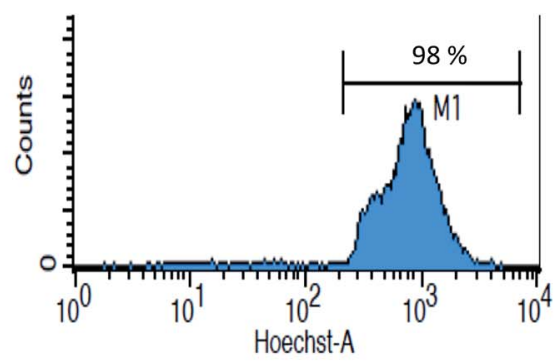

(c)

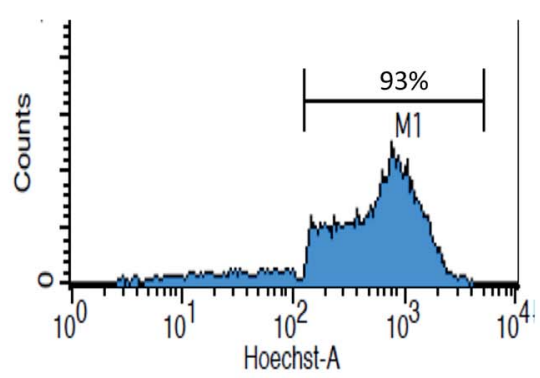

(f)

Fig. 6 Representative dose-dependent capture efficacy by two kinds of Ab MNPs. CD20 positive cells (BCRC 60252) and CD20 negative cells (BCRC 60427) were isolated by using high Ab conjugated or low Ab conjugated MNPs at different concentrations. Panel (a-c): high Ab conjugated MNPs (15.86 $\mu \mathrm{g}$ Ab per mg MNPs). Panel (d-f): low Ab conjugated MNPs (8.07 $\mu \mathrm{g}$ Ab per mg MNPs) at 10, $30,50 \mu \mathrm{g} \mathrm{mL}^{-1} \mathrm{MNPs}$. The flow cytometry data are reported as the mean and standard deviations $(n=2)$. The separation efficacy was $94.2 \pm 1.3 \%$ (a) $96 \pm 0.92 \%$ (b) or $98 \pm$ $0.68 \%$ (c) for high Ab conjugated MNPs. The separation efficacy was $86.5 \pm 2.1 \%$ (d) $94.47 \pm 5.5 \%$ (e) or $93.6 \pm 1.0 \%$ (f) for low Ab conjugated MNPs. 


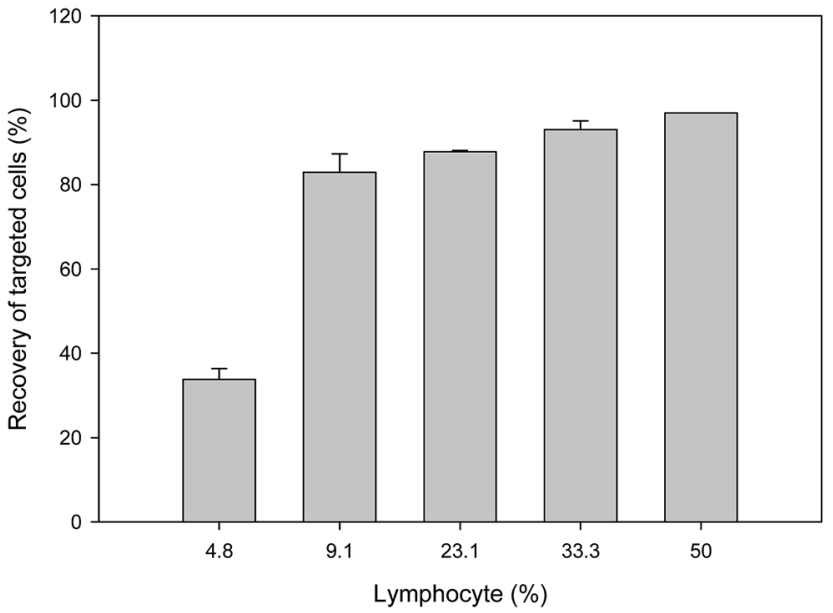

Fig. 7 The isolation efficacy of targeted lymphoma cells from a higher population of hybridoma (CD20 negative) cells by using Ab MNPs. A small number of spiked lymphoma cells ranged from $5 \times 10^{4}$ to $1 \times$ $10^{6}$ were mixed with hybridoma cells fixed at $1 \times 10^{6}$ cells. Each bar represents the standard deviation $(n=2)$.

concentration of $\mathrm{Ab}$ would be necessary to achieve efficient cellular targeting of MNPs. ${ }^{25}$ The above studies showed that both $\mathrm{Ab}$ concentrations (high and low) showed specificity towards the target cells.

Generally, in the early stage of cancer, very few cancer cells are present within a large number of normal cells in the blood. To determine the specificity of Ab MNPs towards less CD20positive lymphocyte cells, the target cells were mixed with non-target cells in different ratios (Fig. 7); stained (Hoechst $33342)$ target cells ratio were varied $\left(5 \times 10^{4}-1 \times 10^{6}\right)$, and stained (Rhodamine B) non-target cells ratio were fixed $(1 \times$ $\left.10^{6}\right)$. The mixed cells were incubated with fixed concentrations $\left(30 \mu \mathrm{g} \mathrm{mL}{ }^{-1}\right.$ ) of Ab MNPs for 20 minutes and then isolated using a magnet and analyzed by flow cytometry. The results showed that even if we used low concentrations of target cells, the $\mathrm{Ab}$ MNPs could recognize the specific target cells against the large population of normal cells. When the target cells were very rare (i.e. only $5 \times 10^{4}$ target lymphocyte cells mixed with $10^{6}$ nontarget cells), only $31 \%$ of target cells were isolated.

\section{Biocompatibility studies}

Factors such as surface conjugation, charges and sizes of MNPS are key aspects in understanding the biocompatibility of the functionalized MNPs. A high concentration of MNPs has been reported to be toxic to in vitro cells due to the generation of reactive oxygen species. ${ }^{28}$ On the contrary, trivial effects on the viability of various cell lines have been reported for these MNPs by other authors. ${ }^{29,30}$ Herein, we evaluated the biocompatibility of modified Ab MNPs using several complementary approaches, such as Trypan blue, cell counting and MTT assay for CD20positive lymphocyte cells. The results of Trypan blue assay and cell counting (Fig. 8a and b) confirmed the biocompatibility of Ab MNPs. The cell viability was above $90 \%$ after 8 hours of incubation. Trypan blue test has been proposed as the standard method to validate cell viability after MNP incubation. The results of cell counting showed that cell density remained the same after 8 hours of incubation with Ab MNPs. The MTT assay (Fig. 8) showed that cell activity was not significantly affected by the presence of $\mathrm{Ab}$ MNPs at 24 hours of treatment ( $>80 \%$ viability in relation to the control sample), even at the highest concentration $\left(50 \mu \mathrm{g} \mathrm{mL} \mathrm{mL}^{-1}\right)$. Moreover, Ab MNPs toxicity effect was also evaluated with normal cell (CHO cells) and presented in Fig. S8. $\dagger$ Higher viability has been observed for the two cell types when compared to the control conditions. MTT assay shows that Ab MNPs do not exert acute adverse effect on CHO cells and lymphoma cells even at high dosage, suggesting that it can be applicable in vivo applications. The MTT assay is based on the reduction of soluble yellow tetrazolium into insoluble purple formazan crystals by mitochondrial succinate dehydrogenase of the viable cell. Thus, the rate of formazan crystal formation is directly proportional to the total number of viable cells, which is measured in terms of absorbance. ${ }^{31} \mathrm{~A}$ modified MTT assay has been adopted here to avoid the influence of MNPs on the colorimetric assay. ${ }^{32}$ Fig. $8 \mathrm{c}$, shows that the viability of cells exhibited a slightly decreasing trend from 1

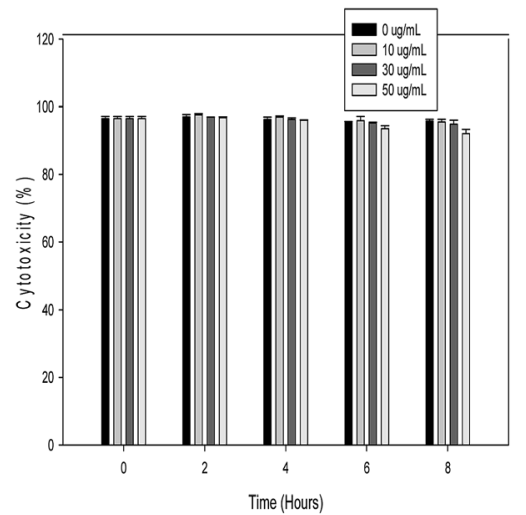

(a)



(b)



(c)

Fig. 8 Biocompatibility of Ab MNPs in lymphoma cell line evaluated by hemocytometer (a) cell counting (b) and MTT assay (c) with different Ab MNPs concentrations ranging from $10-50 \mu \mathrm{gL}^{-1}$ for 8 hours. In the MTT assay, cells with Ab MNPs or COOH MNPs were incubated for 24 hours. The coefficient of variation (SD/mean) is around $4 \%$. 
hour to 24 hours of co-culture for both bare and Ab MNPs in a similar manner to that of the control sample (without MNPs), and the viability remained more than $80 \%$. All the Trypan blue dye, MTT assay, and cell counting results confirmed the compatibility of the Ab MNPs developed in this study. Cell viability could be maintained within the $24 \mathrm{~h}$ incubation using $50 \mu \mathrm{g} \mathrm{mL} \mathrm{L}^{-1}$ of MNPs.

\section{Localization of nanoparticles in the lymphoma cell line}

The Prussian blue staining test was employed to detect the localization of the nanoparticles with antibody-conjugated and unconjugated MNPs on CD20-positive lymphocyte cells (Fig. S9†). This method detects iron within the treated cells through the reduction of ferric ions into the ferrous state, which is shown as a blue colour. ${ }^{33}$ The cells treated with COOH MNPs (antibody free) showed occasional blue spots outside of the cells (Fig. S9a $\dagger$ ), whereas the cells treated with antibody-conjugated nanoparticles showed clear blue shells around the cells, confirming the presence of Ab MNPs around the cell membranes (Fig. S9b $\dagger$ ). There is abundant literature on MNPs conjugation efficiency; however, the results are difficult to compare due to the different experimental protocols, such as size, incubation time, surface coating, longer incubation time and higher concentration. Moreover, longer incubation time and higher concentrations of MNPs enable high interactions with the cell surface and increase the detection efficiency. ${ }^{34}$ High concentrations of MNPs for an extended incubation may reduce viability and enhance cell aggregation. ${ }^{35}$ Therefore, the required quantity of MNPs for cellular uptake must be balanced with their biocompatibility for efficient diagnosis or treatment. ${ }^{29}$ To evaluate the selectivity of Ab MNPs, the iron concentration was also obtained by the potassium thiocyanate method (Fig. 9). In brief, the iron content of different cells $(1 \times$ $10^{6}$ ) was measured after 2 hours of incubation with $30 \mu \mathrm{g}$ of $\mathrm{Ab}$ MNPs or $\mathrm{COOH}$ MNPs at $37{ }^{\circ} \mathrm{C}$. The results showed that a small difference in the amount of accumulated iron in the two cell lines used as controls could be due to either different MNP sizes or the type of cell line. Our potassium thiocyanate data indicated that $\mathrm{Ab}$ MNPs bound effectively at the level of $16.8 \mu \mathrm{g}$ per $10^{-6}$ cells on the cell surface of lymphocytes. In contrast, half the quantity of $\mathrm{Ab}$ MNPs was deposited on CD20-free hybridoma and CHO cells at the level of 7.9 and $8.8 \mu \mathrm{g}$ per $10^{6}$ cells, respectively. Nonspecific iron binding (13-20 $\mu \mathrm{g}$ per $10^{6}$ cells) was observed in the three cell lines when isolation was performed by using $\mathrm{COOH}$ MNPs. This result confirmed the nonspecific binding of $\mathrm{COOH}$ MNPs to cells. Recently, the location of functionalized MNPs in MCF-7 cells after $24 \mathrm{~h}$ incubation has been observed by optical microscopy and TEM. Most of the MNPs were distributed in the periplasmic area. ${ }^{30}$ In addition, their results for Prussian blue staining and ferrozinebased assay also indicated that MNPs ( $21 \mu \mathrm{g}$ per $10^{6}$ cells) can be internalized effectively ( $21 \mu \mathrm{g}$ per $10^{6}$ cells) by MCF-7 cells. In contrast, the major location of our Ab MNPs or COOH MNPs was on the surface of cellular membranes in the lymphoma cells, as indicated in Fig. S9b. $\dagger$ It is important to point out the dose of our MNPs was 13-fold lower than in Calero's paper, which might have resulted in the difference. ${ }^{30}$

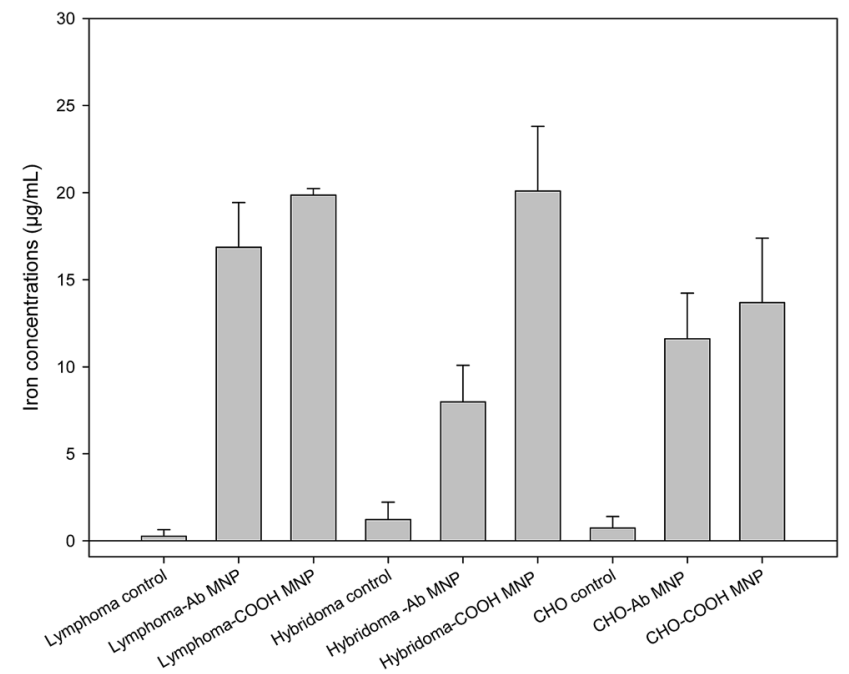

Fig. 9 Quantification of intracellular iron content. Thiocyanate-based iron uptake among the cell lines. The three cell lines were incubated with $30 \mu \mathrm{g} \mathrm{mL}{ }^{-1}$ of Ab MNPs or $\mathrm{COOH}$ MNPs at $37^{\circ} \mathrm{C}$ for 2 hours. The excess iron was washed with PBS, and the iron concentration in $1 \times$ $10^{6}$ cells from each cell line was determined using a thiocyanatebased spectrophotometric assay. The error bars in the figure represent the mean and standard deviation (SD) of four repeats.

\section{Conclusion}

In this study, we demonstrated a simple and robust conjugation method by using antibodies and MNPs. The specificity for targeting the thiols of the Fc fragment in an antibody can be improved by using maleimide conjugation. The synthetic platform described herein was efficient. It has the potential for further optimization for antibody conjugation on different nanoparticles. In addition, the lymphocytes cells were successfully detected and isolated from mixed samples containing other cells and target lymphocytes cells. The Ab MNPs fully retained their antibody binding capacity and could detect and isolate the CD20-positive cells from the mixed cells. The capture efficiency of Ab MNPs for lymphoma cells was above 95\% under our experimental conditions. The capture efficiency was confirmed by complementary techniques, such as flow cytometry and confocal microscopy. Based on this simple antibody conjugation on MNPs, this platform enables robust cell sorting by using only a permanent magnet. The present approach demonstrated a facile, time saving and economical synthesis of Ab MNPs that can be used as an efficient capture platform for tumour cells.

\section{Conflict of interest}

The authors would like to declare that no potential conflicts of interest exist.

\section{Acknowledgements}

We express gratitude to Ministry of Science and Technology (MOST 105-2221-E-182-070), Chang Gung University (BMRP 
758) and Chang Gung Memorial Hospital (CMRPD 1E0352) for funding and supporting this research.

\section{References}

1 M. Yu, S. Stott, M. Toner, S. Maheswaran and D. A. Haber, Circulating tumor cells: approaches to isolation and characterization, J. Cell Biol., 2011, 192, 373-382.

2 C. Wyatt Shields Iv, C. D. Reyes and G. P. López, Microfluidic cell sorting: a review of the advances in the separation of cells from debulking to rare cell isolation, Lab Chip, 2015, 15, 1230-1249.

3 Z. Bakhtiary, A. A. Saei, M. J. Hajipour, M. Raoufi, O. Vermesh and M. Mahmoudi, Targeted superparamagnetic iron oxide nanoparticles for early detection of cancer: possibilities and challenges, Nanomedicine: Nanotechnology, Biology and Medicine, 2016, 12, 287-307.

4 D. Guldner, J. K. Hwang, M. C. D. Cardieri, M. Eren, P. Ziaei, M. G. Norton, et al., In vitro evaluation of the biological responses of canine macrophages challenged with PLGA nanoparticles containing monophosphoryl lipid A, PLoS One, 2016, 11(11), e0165477.

5 S. H. Huang and R. S. Juang, Biochemical and biomedical applications of multifunctional magnetic nanoparticles: a review, J. Nanopart. Res., 2011, 13, 4411-4430.

6 H. Xu, Z. P. Aguilar, L. Yang, M. Kuang, H. Duan, Y. Xiong, et al., Antibody conjugated magnetic iron oxide nanoparticles for cancer cell separation in fresh whole blood, Biomaterials, 2011, 32, 9758-9765.

7 M. A. Firer and G. Gellerman, Targeted drug delivery for cancer therapy: the other side of antibodies, J. Hematol. Oncol., 2012, 5, 1-16.

8 S. H. Lim, S. A. Beers, R. R. French, P. W. M. Johnson, M. J. Glennie and M. S. Cragg, Anti-CD20 monoclonal antibodies: historical and future perspectives, Haematologica, 2010, 95, 135-143.

9 L. Al-Zoobi, S. Salti, A. Colavecchio, M. Jundi, A. Nadiri, G. S. Hassan, et al., Enhancement of rituximab-induced cell death by the physical association of CD20 with CD40 molecules on the cell surface, Int. Immunol., 2014, 26, 451465.

10 P. Jain and S. O'Brien, Anti-CD20 monoclonal antibodies in chronic lymphocytic leukemia, Expert Opin. Biol. Ther., 2013, 13, 169-182.

11 F. M. Kievit, Z. R. Stephen, O. Veiseh, H. Arami, T. Wang, V. P. Lai, et al., Targeting of primary breast cancers and metastases in a transgenic mouse model using rationally designed multifunctional SPIONs, ACS Nano, 2012, 6, 2591-2601.

12 H. J. Hathaway, K. S. Butler, N. L. Adolphi, D. M. Lovato, R. Belfon, D. Fegan, et al., Detection of breast cancer cells using targeted magnetic nanoparticles and ultra-sensitive magnetic field sensors, Breast Cancer Res., 2011, 13, R108.

13 A. Cirstoiu-Hapca, L. Bossy-Nobs, F. Buchegger, R. Gurny and F. Delie, Differential tumor cell targeting of anti-HER2 (Herceptin ${ }^{\circledR}$ ) and anti-CD20 (Mabthera ${ }^{\circledR}$ ) coupled nanoparticles, Int. J. Pharm., 2007, 331, 190-196.
14 E. Q. Song, J. Hu, C. Y. Wen, Z. Q. Tian, X. Yu, Z. L. Zhang, et al., Fluorescent-magnetic-biotargeting multifunctional nanobioprobes for detecting and isolating multiple types of tumor cells, ACS Nano, 2011, 5, 761-770.

15 F. M. Kievit, Z. R. Stephen, O. Veiseh, H. Arami, T. Wang, V. P. Lai, et al., Targeting of primary breast cancers and metastases in a transgenic mouse model using rationally designed multifunctional SPIONs, ACS Nano, 2012, 6, 2591-2601.

16 S.-M. Jo, S.-H. Noh, Z. Jin, Y. Lim, J. Cheon and H.-S. Kim, Simple and efficient capture of EGFR-expressing tumor cells using magnetic nanoparticles, Sens. Actuators, B, 2014, 201, 144-152.

17 J. Wu, X. Wei, J. Gan, L. Huang, T. Shen, J. Lou, et al., Multifunctional Magnetic Particles for Combined Circulating Tumor Cells Isolation and Cellular Metabolism Detection, Adv. Funct. Mater., 2016, 26, 4016-4025.

18 G. T. Hermanson, 8-Tags and Probes. Bioconjugate Techniques, Academic Press, San Diego, 1996, pp. 297-416.

19 S. H. Tseng, M. Y. Chou and I. M. Chu, Cetuximabconjugated iron oxide nanoparticles for cancer imaging and therapy, Int. J. Nanomed., 2015, 10, 3663-3685.

20 Y.-C. Lu, P.-C. Luo, C.-W. Huang, Y.-L. Leu, T.-H. Wang, K.-C. Wei, et al., Augmented cellular uptake of nanoparticles using tea catechins: effect of surface modification on nanoparticle-cell interaction, Nanoscale, 2014, 6, 10297-10306.

21 F. Rusmini, Z. Zhong and J. Feijen, Protein immobilization strategies for protein biochips, Biomacromolecules, 2007, 8, 1775-1789.

22 M. Wilchek, E. A. Bayer and O. Livnah, Essentials of biorecognition: the (strept)avidin-biotin system as a model for protein-protein and protein-ligand interaction, Immunol. Lett., 2006, 103, 27-32.

23 S. Sun, M. Ma, N. Qiu, X. Huang, Z. Cai, Q. Huang, et al., Affinity adsorption and separation behaviors of avidin on biofunctional magnetic nanoparticles binding to iminobiotin, Colloids Surf., B, 2011, 88, 246-253.

24 R. Rezaeipoor, R. John, S. G. Adie, E. J. Chaney, M. Marjanovic, A. L. Oldenburg, et al., Fc-directed antibody conjugation of magnetic nanoparticles for enhanced molecular targeting, J. Innovative Opt. Health Sci., 2009, 2, 387-396.

25 L. Nobs, F. Buchegger, R. Gurny and E. Allémann, Biodegradable nanoparticles for direct or two-step tumor immunotargeting, Bioconjugate Chem., 2006, 17, 139-145.

26 K. Yan, P. Li, H. Zhu, Y. Zhou, J. Ding, J. Shen, et al., Recent advances in multifunctional magnetic nanoparticles and applications to biomedical diagnosis and treatment, $R S C$ Adv. , 2013, 3, 10598-10618.

27 A. Cirstoiu-Hapca, L. Bossy-Nobs, F. Buchegger, R. Gurny and F. Delie, Differential tumor cell targeting of anti-HER2 (Herceptin) and anti-CD20 (Mabthera) coupled nanoparticles, Int. J. Pharm., 2007, 331, 190-196.

28 A. Hanini, A. Schmitt, K. Kacem, F. Chau, S. Ammar and J. Gavard, Evaluation of iron oxide nanoparticle biocompatibility, Int. J. Nanomed., 2011, 6, 787-794. 
29 M. Calero, L. Gutiérrez, G. Salas, Y. Luengo, A. Lázaro, P. Acedo, et al., Efficient and safe internalization of magnetic iron oxide nanoparticles: two fundamental requirements for biomedical applications, Nanomedicine: Nanotechnology, Biology and Medicine, 2014, 10, 733-743.

30 M. Calero, M. Chiappi, A. Lazaro-Carrillo, M. J. Rodriguez, F. J. Chichon, K. Crosbie-Staunton, et al., Characterization of interaction of magnetic nanoparticles with breast cancer cells, J. Nanobiotechnol., 2015, 13, 16.

31 S. Shukla, A. Jadaun, V. Arora, R. K. Sinha, N. Biyani and V. K. Jain, In vitro toxicity assessment of chitosan oligosaccharide coated iron oxide nanoparticles, Toxicol. Rep., 2015, 2, 27-39.

32 S. Laurent, C. Burtea, C. Thirifays, U. O. Häfeli and M. Mahmoudi, Crucial ignored parameters on nanotoxicology: the importance of toxicity assay modifications and "cell vision", PLoS One, 2012, 7, e29997.

33 M. Neri, C. Maderna, C. Cavazzin, V. Deidda-Vigoriti, L. S. Politi, G. Scotti, et al., Efficient in vitro labeling of human neural precursor cells with superparamagnetic iron oxide particles: relevance for in vivo cell tracking, Stem Cells, 2008, 26, 505-516.

34 T. Schlorf, M. Meincke, E. Kossel, C. C. Gluer, O. Jansen and R. Mentlein, Biological properties of iron oxide nanoparticles for cellular and molecular magnetic resonance imaging, Int. J. Mol. Sci., 2010, 12, 12-23.

35 S. Naqvi, M. Samim, M. Abdin, F. J. Ahmed, A. Maitra, C. Prashant, et al., Concentration-dependent toxicity of iron oxide nanoparticles mediated by increased oxidative stress, Int. J. Nanomed., 2010, 5, 983-989. 